\title{
Contrast Sensitivity and Eye Drops
}

\author{
Theodosios Chatzibalis ${ }^{1}$, Konstantinos Stamoulas ${ }^{1}$, Athanasios Karamitsos ${ }^{1}$, Diamantis Almaliotis ${ }^{1}$, \\ Vasiliki Mirtsou-Fidani ${ }^{2}$, Nikolaos Georgiadis ${ }^{3}$, Vasileios Karampatakis ${ }^{1}$
}

${ }^{1}$ Laboratory of Experimental Ophthalmology, Medical School, Aristotle University of Thessaloniki, Thessaloniki, Greece; ${ }^{2}$ Laboratory of Pharmacology, Medical School, Aristotle University of Thessaloniki, Thessaloniki, Greece; ${ }^{3} 1$ st Eye Clinic, Medical School, Aristotle University of Thessaloniki, Thessaloniki, Greece.

Email: almaliotis_diamantis@yahoo.gr

Received March $13^{\text {th }}, 2012$; revised April 16 $6^{\text {th }}, 2012$; accepted May $10^{\text {th }}, 2012$

\begin{abstract}
Purpose: To evaluate the influence of commonly used eye drops on contrast sensitivity (CS). Design: Prospective study. Participants: One hundred twenty volunteers were enrolled. Methods: The CS of ophthalmologically healthy individuals was evaluated at baseline examination before the instillation, 20 and 40 minutes after the instillation of diclofenac sodium and commonly used anti-glaucoma eye drops (latanoprost, brimonidine tartrate, combination of dorzolamide hydrochloride and timolol maleate). CS was assessed at 7 spatial frequencies varying from $1.5 \mathrm{c} / \mathrm{d}$ to $20 \mathrm{c} / \mathrm{d}$ by the use of Mentor B-VAT II-SG video acuity tester. Results: Diclofenac sodium induced a mild, however statistically significant decrease on the CS in 4 out of 30 subjects 20 minutes after instillation at the spatial frequency of $1.5 \mathrm{c} / \mathrm{d}$ and 3 $\mathrm{c} / \mathrm{d}(\mathrm{p}<0.05$ for both frequencies). Latanoprost induced a decrease in CS in 1 out of 30 subjects and combination of dorzolamide hydrochloride and timolol maleate in 2 out of 30 at low spatial frequencies $(1.5 \mathrm{c} / \mathrm{d}$ and $3 \mathrm{c} / \mathrm{d}) 20$ minutes after the instillation and this decrease was not statistically significant for any of these drugs $(\mathrm{p}>0.05)$. Brimonidine tartrate decreased CS in 4 out of 30 subjects 20 minutes after the instillation at a high spatial frequency $(20 \mathrm{c} / \mathrm{d})$ yet this decrease was marginally statistically significant $(p=0.057)$. CS returned to baseline scores 40 minutes after the instillation for all drugs. Conclusions: Diclofenac sodium eye drops had a mild temporal effect on the low spatial frequencies of $1.5 \mathrm{c} / \mathrm{d}$ and $3 \mathrm{c} / \mathrm{d}$ in some individuals. Latanoprost and dorzolamide/timolol had a non-statistically significant effect on the same spatial frequencies. Brimonidine eye drops had a temporal effect on the high spatial frequency of $20 \mathrm{c} / \mathrm{d}$ with marginal statistical significance. The CS scores returned to normal, in all cases, within the next 40 minutes after the instillation. Ophthalmologists should be aware of the fact that some eye drops may have an influence on CS.
\end{abstract}

Keywords: Cornea; Ocular Surface; External Disease

\section{Introduction}

Despite the growing number of drugs used as anti-inflammatory agents in ophthalmology, diclofenac remains a reasonable choice when treating a variety of ocular conditions. Owing its anti-inflammatory activity to cycloxygenase inhibition which in turn leads to reduction in prostaglandin synthesis, this non-steroidal anti-inflammatory drug (NSAID) is commonly used for prophylaxis and treatment of postoperative inflammation. Yet diclofenac exhibits certain adverse effects after topical instillation, burning and stinging being the most frequent [1]. Blurred vision has also been reported.

Topical beta-blockers, carbonic anhydrase inhibitors such as dorzolamide, latanoprost, usually in combination with beta-blockers, and brimonidine, may have various side effects, among them blurring of vision.

CS is a parameter used to assess the visual function at different levels of contrast, usually altered before any severe change in visual acuity is noticed [2]. There are various studies correlating the ability of individuals to fulfill complex tasks, such as driving performance [3] or computer task accuracy [4], or even accomplish everyday activities with their CS function. A decrease in the quality of life has also been shown in relation to the deterioration of visual function [5], including CS.

Because all of the aforementioned drugs included in our study are widely used in every day practice and some patients report vision blurring after their instillation, the purpose of the present study was to evaluate the effect that these drugs have on CS and consequently on the quality of vision.

\section{Materials and Methods}

The aim of this study was to evaluate the blurring of vision, which is related to the instillation of commonly used eye drops. 
Healthy volunteers without any ocular disease were recruited for this study. Personal medical history was recorded and all subjects were free of medication. Individuals reporting symptoms of dry eye or using any other ophthalmic eye drops (natural tears included) and contact lenses were excluded from the study. Written informed consent was obtained from each participant. After a short explanation of the procedure about to be followed subjects were examined by an ophthalmologist under a slit lamp. Individuals with abnormal findings from the anterior segment or the fundus were also excluded. All individuals had Snellen visual acuity of 10/10.

A total of one hundred and twenty volunteers were eligible for inclusion and were finally enrolled in the study. All participants had best-corrected visual acuity of 10/10 or better. The study adhered to the tenets of the Declaration of Helsinki and the procedure followed a standard protocol that was consistent across the examination.

The participants were randomly divided in four groups. Each group consisted of 30 subjects, 15 males and 15 females. The CS function of each subject was assessed with the Mentor B-VAT II-SG video acuity tester (Mentor O \& O, Norwell, Mass). This monitor has been used for the evaluation of visual function in several conditions $[6,7]$. Apart from its other features, the monitor uses sinusoidal gratings of different spatial frequencies and contrast levels for CS function measurements. In specific, sixteen spatial frequencies are available from $1.5 \mathrm{c} / \mathrm{d}$ to $40 \mathrm{c} / \mathrm{d}$ yet we examined the CS function of our subjects at $1.5 \mathrm{c} / \mathrm{d}, 3.0 \mathrm{c} / \mathrm{d}, 4.6 \mathrm{c} / \mathrm{d}, 6.0 \mathrm{c} / \mathrm{d}, 8.5 \mathrm{c} / \mathrm{d}, 12 \mathrm{c} / \mathrm{d}$ and 20 $\mathrm{c} / \mathrm{d}$. For every spatial frequency there are available thirty two contrast levels varying from $98 \%$ to $0.1 \%$ of contrast. From $98 \%$ to $16 \%$ contrast levels can be changed in steps of $0.1 \log$ CS units whilst from $10 \%$ to $0.1 \%$ contrast level the $\log$ CS units are allocated at regular intervals of $0.2 \log$ units.

The psychophysical method used was the three alternative forced choice. Subjects were left to adapt to mesopic conditions wearing their best-spectacle correction. The test luminance was automatically calibrated to 85 $\mathrm{cd} / \mathrm{m}^{2}$ and the sinusoidal gratings were presented by the examiner for approximately 5 seconds using a hand held controller. Grating's orientation could be vertical, $-14^{\circ}$ to the left or $+14^{\circ}$ to the right from vertical. The orientation varied randomly between the trials. The measurements advanced gradually from a higher to a lower contrast level. At each level the subject was asked to identify the grating's orientation at four different trials. When three out of four responses were correct (at random sequence) the contrast level was considered to be passed and the examiner advanced to a lower one. The last contrast level they passed was defined as the contrast threshold of the eye and was recorded. The measurements obtained were converted to $\log \mathrm{CS}$ for the statistical analysis. The test was performed at a 3-meter distance.

CS was recorded for both eyes at baseline examination before the instillation of the drugs.

After an initial assessment of CS in the 1st group of patients (mean age $30.83 \pm 10.13$ years) diclofenac sodium eye drops were instilled, in the 2 nd group (mean age $31.73 \pm 10.95$ years) latanoprost eye drops, in the 3 rd group (mean age $32.60 \pm 10.08$ years) dorzolamide hydrochloride/timolol maleate and in the 4th group (mean age $32.43 \pm 8.86$ years) brimonidine tartrate.

After the baseline CS examination the test was repeated 20 and 40 minutes after the instillation.

The data obtained were analyzed using paired-samples $t$-test to assess the CS changes 20 minutes after the instillation, given that the scores returned to baseline levels 40 minutes after the drug instillation for all groups and at any spatial frequency. All statistical analyses were performed using SPSS version 18.0 software (SPSS Inc., Chicago, IL); $\mathrm{p}<0.05$ was judged as statistically significant.

\section{Results}

The log CS was recorded for all groups 20 and 40 minutes after the eye drop instillation.

As regards the first group (diclofenac sodium), a mild CS decrease by $0.2 \log$ units was observed in 4 out of 30 subjects (13.33\%) 20 minutes after the instillation at spatial frequencies of $1.5 \mathrm{c} / \mathrm{d}$ and $3 \mathrm{c} / \mathrm{d}(\mathrm{p}<0.05, \mathrm{p}=0.043$, paired samples $t$-test).

Concerning the second group (latanoprost), $\log$ CS was decreased by $0.2 \log$ units in only 1 out of 30 subjects $(3.33 \%) 20$ minutes after the instillation at spatial frequencies of $1.5 \mathrm{c} / \mathrm{d}$ and $3 \mathrm{c} / \mathrm{d}$. This change was not statistically significant $(\mathrm{p}=0.326$, paired samples $t$-test).

In the third group (dorzolamide/timolol) 2 out of 30 subjects $(6.66 \%)$ showed also a decrease in CS by $0.2 \mathrm{log}$ units at spatial frequencies of $1.5 \mathrm{c} / \mathrm{d}$ and $3 \mathrm{c} / \mathrm{d} 20 \mathrm{~min}-$ utes after the instillation. Though this change was not found to be statistically significant $(p=0.161$, paired samples $t$-test) in the group of the examined subjects.

In the fourth group (brimonidine) 4 out of 30 subjects (13.33\%) showed a decrease in CS by 0.2 log units (in one subject $0.4 \log$ units) 20 minutes after the eye drop instillation at the spatial frequency of $20 \mathrm{c} / \mathrm{d}$. The recorded scores were approaching a statistically significant difference at this time point $(\mathrm{p}=0.057$, paired samples $t$-test).

No change was observed at the other spatial frequencies examined for any of the eye drops instilled. Concerning the spatial frequencies in which a change was recorded, all scores returned to baseline examination levels $40 \mathrm{mi}-$ nutes after the instillation for all of the drugs used. 
Table 1. Summary of contrast sensitivity (CS) changes.

\begin{tabular}{lccc}
\hline \multicolumn{1}{c}{ Regimen } & $\begin{array}{c}\text { Number of subjects } \\
\text { with CS impairment }\end{array}$ & $\begin{array}{c}\text { Percentage }(\%) \\
\text { of affected }\end{array}$ & $\begin{array}{c}\text { Altered spatial } \\
\text { frequencies }\end{array}$ \\
\hline Diclofenac sodium & 4 out of 30 & 13.33 & $1.5 \mathrm{c} / \mathrm{d}$ and $3 \mathrm{c} / \mathrm{d}(0.2 \mathrm{log}$ units $)$ \\
\hline Latanoprost & 1 out of 30 & 3.33 & $1.5 \mathrm{c} / \mathrm{d}$ and $3 \mathrm{c} / \mathrm{d}(0.2 \mathrm{log} \mathrm{units})$ \\
\hline Dorzolamide/Timolol & 2 out of 30 & 6.66 & $1.5 \mathrm{c} / \mathrm{d}$ and $3 \mathrm{c} / \mathrm{d}(0.2 \mathrm{log}$ units $)$ \\
\hline Brimonidine & 4 out of 30 & 13.33 & $20 \mathrm{c} / \mathrm{d}(0.2 \log$ units, in one subject $0.4 \log$ units $)$ \\
\hline
\end{tabular}

$\mathrm{c} / \mathrm{d}=$ cycles/degree.

All results are summarized in Table 1.

\section{Discussion}

CS function has been used to evaluate the quality of vision in several conditions including various forms of retinopathy [8], cataract surgery/IOL implantation [9] and glaucoma [10]. The effect of a variety of drugs on visual function has also been studied [11-13]. As regards glaucoma, most studies have focused on the long term effect of anti-glaucoma eye drops on visual function. Their results remain controversial although there is a great number of reports outlining an improvement in CS four or more weeks after the drug administration, especially in intermediate spatial frequencies $(6 \mathrm{c} / \mathrm{d}$ and 12 c/d) [12]. This improvement can be attributed to a recovery of the damaged ganglion cells and their microenvironment due to the lowering of the IOP and an increase in the ocular perfusion and haemodynamics [14]. In the pre- sent study we investigated the effect of commonly used anti-glaucoma drugs on CS immediately after the drug instillation. To the best of our knowledge there is only one report concerning the influence of anti-glaucoma drugs on visual quality immediately after the drug ad- ministration [15]. In this report the investigators found a substantial reduction in CS function for at least 5 minutes after the instillation of either timolol gel-forming solution or brinzolamide. This temporary decrease was fully re- stored 15 minutes after the drug instillation. In our study the effect of the drugs was examined 20 and 40 minutes after the drug instillation and we used timolol only in combination with dorzolamide. As concerns diclofenac sodium, there are reports underlining an improvement on CS after cataract surgery one month after the drug ad- ministration [16]. In the present study the decrease of CS function was detected only immediately after the instilla- tion.

Furthermore, West et al. [17] have showed that a CS level of $\leq 1.40 \log$ units and $\leq 1.30 \log$ units are associated to disability in reading and recognition of faces respectively in people older than 65 years of age. In our study a mild decrease was recorded only in 2 low spatial frequencies $(1.5 \mathrm{c} / \mathrm{d}$ and $3 \mathrm{c} / \mathrm{d})$ after the instillation of di- clofenac sodium. This reduction was $0.20 \mathrm{log}$ units in every of the four subjects that manifested the decrease on their visual function. As regards the anti-glaucoma drops (latanoprost, dorzolamide/timolol) used in our study, although no statistically significant decrease was observed at any of the spatial frequencies tested, in some individuals a mild reduction by $0.2 \log$ units was recorded also in the low spatial frequencies of $1.5 \mathrm{c} / \mathrm{d}$ and $3.0 \mathrm{c} / \mathrm{d}$. Consequently some patients may experience a mild visual disturbance under low luminance conditions. Only brimonidine had an influence on the high spatial frequency of $20 \mathrm{c} / \mathrm{d},(\mathrm{p}=0.057$, marginal statistical significance) and consequently some patients may have a mild and temporal effect on the quality of vision.

Though the number of subjects recruited in the present study is not very large, the results are indicative of the visual disturbance that some individuals may experience after the instillation of the above eye drops.

In conclusion, the CS was temporarily influenced in some individuals. The impaired CS scores returned to the pre-instillation status within the next 40 minutes. Ophthalmologist should be aware that CS impairment may be a potential cause of visual disturbances, under various luminance conditions, after the instillation of the above eye drops.

\section{REFERENCES}

[1] A. J. Flach, "Topical Nonsteroidal Anti-Inflammatory Drugs in Ophthalmology," International Ophthalmology Clinics, Vol. 42, No. 1, 2002, pp. 1-11. doi:10.1097/00004397-200201000-00003

[2] A. S. Hawkins, J. P. Szlyk, Z. Ardickas, K. R. Alexander and J. T. Wilensky, "Comparison of Contrast Sensitivity, Visual Acuity, and Humphrey Visual Field Testing in Patients with Glaucoma," Journal of Glaucoma, Vol. 12, No. 2, 2003, pp. 134-138. doi:10.1097/00061198-200304000-00008

[3] C. Owsley, K. Ball, G. McGwin Jr., et al., "Visual Processing Impairment and Risk of Motor Vehicle Crash among Older Adults," Journal of the American Medical Association, Vol. 279, No. 14, 1998, pp. 1083-1088.

[4] I. U. Scott, W. J. Feuer and J. A. Jacko, "Impact of Visual Function on Computer Task Accuracy and Reaction Time 
in a Cohort of Patients with Age-Related Macular Degeneration," American Journal of Ophthalmology, Vol. 133, No. 3, 2002, pp. 350-357. doi:10.1016/S0002-9394(01)01406-4

[5] G. C. Brown, "Vision and Quality-of-Life," Transactions of the American Ophthalmological Society, Vol. 97, 1999, pp. 473-511.

[6] D. Eisenmann, R. Wagner, B. Dick and K. W. Jacobi, "Effect of Corneal Astigmatism on Contrast Sensitivity in Mono- And Multifocal Pseudophakia-A Theoretical Study of the Physical Eye," Klin Monbl Augenheilkd, Vol. 209, No. 2-3, 1996, pp.125-131. doi:10.1055/s-2008-1035291

[7] K. R. Alexander, D. J. Derlacki and G. A. Fishman, "Contrast Thresholds for Letter Identification in Retinitis Pigmentosa," Investigative Ophthalmology \& Visual Science, Vol. 33, No. 6, 1992, pp. 1846-1852.

[8] G. Trick, R. Burde, M. Gordon, J. Santiago and C. Kilo, "The Relationship between Hue Discrimination and Contrast Sensitivity Deficits in Patients with Diabetes Mellitus," Ophthalmology, Vol. 95, No. 5, 1988, pp. 693-698.

[9] J. Jay, H. Chakrabarti and J. Morrison, "Quality of Vision through Diffractive Bifocal Intraocular Lenses," British Journal of Ophthalmology, Vol. 75, No. 6, 1991, pp. 359366. doi:10.1136/bjo.75.6.359

[10] R. L. Stamper, "Psychophysical Changes in Glaucoma," Survey of Ophthalmology, Vol. 33, 1989, pp.309-318.

[11] B. E. Klein, R. Klein, M. D. Knudtson, K. E. Lee, L. G. Danforth, J. O. Reinke and A. M. Adler, "Associations of Selected Medications and Visual Function: The Beaver Dam Eye Study," British Journal of Ophthalmology, Vol. 87, No. 4, 2003, pp. 403-408. doi:10.1136/bjo.87.4.403
[12] T. S. Prata, M. V. Piassi and L. Melo Jr., "Changes in Visual Function after Intraocular Pressure Reduction Using Antiglaucoma Medications," Eye, Vol. 23, 2009, pp. 1081-1085. doi:10.1038/eye.2008.226

[13] O. Arend, A. Harris, P. Wolter and A. Remky, "Evaluation of Retinal Haemodynamics and Retinal Function after Application of Dorzolamide, Timolol and Latanoprost in Newly Diagnosed Open-Angle Glaucoma Patients," Acta Ophthalmologica Scandinavica, Vol. 81, No. 5, 2003, pp. 474-479. doi:10.1034/j.1600-0420.2003.00122.x

[14] D. W. Evans, S. L. Hosking, D. Gherghel and J. D. Bartlett, "Contrast Sensitivity Improves after Brimonidine Therapy in Primary Open Angle Glaucoma: A Case for Neuroprotection," British Journal of Ophthalmology, Vol. 87, No. 12, 2003, pp. 1463-1465. doi:10.1136/bjo.87.12.1463

[15] T. Hiraoka, D. Masakazu, O. Fumiki, K. Takahiro and O. Tetsuro, "Contrast Sensitivity and Optical Quality of the Eye after Instillation of Timolol Maleate Gel-Forming Solution and Brinzolamide Ophthalmic Suspension," Ophthalmology, Vol. 117, No. 11, 2010, pp. 2080-2087. doi:10.1016/j.ophtha.2010.03.028

[16] L. Rossetti, E. Bujtar, D. Castoldi, C. Torrazza and N. Orzalesi, "Effectiveness of Diclofenac Eyedrops in Reducing Inflammation and the Incidence of Cystoid Macular Edema after Cataract Surgery," Journal of Cataract \& Refractive Surgery, Vol. 22, No. 1, 1996, pp. 794-799.

[17] S. K. West, G. S. Rubin, A. T. Broman, B. Munoz, K. Bandeen-Roche and K. Turano, "How Does Visual Impairement Affect Performance on Tasks of Everyday Life," Archives of Ophthalmology, Vol. 120, No. 6, 2002, pp. 774-780. doi:10.1001/archopht.120.6.774 